\title{
Telmisartán, ramipril y su combinación en pacientes de alto riesgo vascular
}

\author{
Telmisartan, ramipril and both in patients at high risk for vascular Events
}

The Ontarget Investigators N Engl J Med 2008;358:1547-59

\section{Objetivo}

EComparar la eficacia de ramipril, telmisartán y su combinación para prevenir eventos cardio-vasculares (CV) mayores en pacientes con enfermedad CV o diabetes de alto riesgo.

\section{Diseño y lugar}

Ensayo clínico aleatorizado doble ciego (ECCA) con 5,5 años de seguimiento, en 733 centros de 40 países.

\section{Pacientes}

Mayores de 55 años con enfermedad coronaria, arterial periférica o cerebrovascular o diabetes con daño de órgano blanco.

\section{Intervención}

Luego de un período de "run-in"* que incluyó a 29018 sujetos, 8576 fueron aleatorizados a recibir $10 \mathrm{mg} /$ día de ramipril, 8542 a $80 \mathrm{mg} /$ día de telmisartán y 8502 a tratamiento combinado.

\section{Medición de resultados principales}

Muerte CV, infarto agudo de miocardio (IAM) accidente cerebrovascular (ACV) u hospitalización por insuficiencia cardíaca (IC).

\section{Resultados principales}

Respecto del grupo ramipril, la presión arterial (PA) promedio fue menor en el grupo telmisartán $(-0,9 /-0,6 \mathrm{mmHg})$ y en el de tratamiento combinado $(-2,4 /-1,4 \mathrm{mmHg})$. No hubo diferencias en el resultado primario pero si en las causas de abandono. Ver tabla 1.

Tabla 1: resultado primario y causas de abandono.

\begin{tabular}{|c|c|c|c|c|c|}
\hline Resultados & $\begin{array}{c}\text { Ramipril(R) } \\
n=8576\end{array}$ & $\underset{\mathrm{n}=8542}{\text { Telmisartán(T) }}$ & $\begin{array}{c}\text { Ambos(A) } \\
n=8502\end{array}$ & \multicolumn{2}{|c|}{\begin{tabular}{|c|c|} 
T us. R & A vs. \\
$\begin{array}{c}\text { Significancia } \\
\text { estadstica (p) }\end{array}$
\end{tabular}} \\
\hline Resultado primario $^{\mathrm{a}}$ & $1412(16,5 \%$ & $1423(16,7 \%)$ & $1386(16,3 \%$ & & NS \\
\hline Abandonos totales & $2099(24,5 \%$ & $1962(23,0 \%)$ & $2495(29,3 \%)$ & 0,02 & 0,001 \\
\hline Causa de Sintomas hipotensivos & $149(1,7 \%)$ & $229(2,7 \%)$ & $406(4,8 \%)$ & 0,001 & $1 \mid 0,001$ \\
\hline abandono Síncope & $15(0,2 \%)$ & $19(0,2 \%)$ & $29(0,3 \%)$ & NS & 0,03 \\
\hline Tos & $360(4,2 \%)$ & $93(1,1 \%)$ & $392(4,6 \%)$ & 0,001 & NS \\
\hline Angioedema & $25(0,3 \%)$ & $10(0,1 \%)$ & $18(0,2 \%)$ & 0,01 & NS \\
\hline Deterioro renal & $60(0,7 \%)$ & $68(0,8 \%)$ & $94(1,1 \%)$ & NS & 0,001 \\
\hline
\end{tabular}

auerte de causa cardiovascular, infarto agudo de miocardio, accidente cerebrovascular u hospitalización por insuficiencia cardíaca.

\section{Conclusiones}

El telmisartán fue equivalente a ramipril en pacientes con enfermedad CV o diabetes de alto riesgo y se asoció con menos tos y angioedema. La combinación de ambas drogas incrementó los eventos adversos pero no los beneficios.

Palabras clave: losartán, ramipril, riesgo cardiovascular.

Key words: losartan, ramipril, cardiovascular risk.

Fuente de financiamiento: Boehringer-Ingelheim

\section{Comentario}

En pacientes con enfermedad CV o diabetes de alto riesgo, los inhibidores de la enzima de conversión de angiotensina (IECA) ya habían demostrado reducir la morbimortalidad CV pero era desconocido el papel de los antagonistas de los receptores de la angiotensina II (ARAII).

Este ECCA de "no inferioridad" de alta calidad demuestra que $80 \mathrm{mg} /$ día de telmisartán preservan el 95\% (IC95\% 83 a 106) del beneficio en el resultado primario de $10 \mathrm{mg} /$ día de ramipril vs. placebo, como ya se había comunicado en el estudio HOPE'. Casi con el mismo diseño, el estudio VALIANT había documentado que el $320 \mathrm{mg} /$ día de valsartán en dos tomas diarias preserva el 100\% (IC95\% 60 a 139) del beneficio atribuido a tres tomas diarias de $50 \mathrm{mg}$ de captopril, como se había demostrado en un meta-análisis de tres ensayos clínicos en pacientes con $\mathrm{IAM}^{2}$.

En otro ensayo clínico (OPTIMAAL) no fue confirmada la "no inferioridad" de $50 \mathrm{mg}$ diarios de losartán respecto de captopril $50 \mathrm{mg}$, tres veces al día ${ }^{3}$. Por otro lado, en pacientes con IC el doble bloqueo del sistema renina angiotensina (Val.HEFT y CHARM aditivo) se asoció a una reducción en el riesgo de internaciones por IC y a la mortalidad CV ${ }^{4-6}$.

\section{Conclusiones del comentador}

En los pacientes que recibieron telmisartán y valsartán en los estudios ONTARGET y VALIANT respectivamente, hubo una similar o aún mayor reducción de la PA, mas episodios hipotensivos y mayor alteración de la función renal, lo que estaría relación con un efecto en "J" de la PA en pacientes con enfermedad CV previa y/o con enfermedad renovascular bilateral inadvertida lo que implicaría ausencia de beneficio con el doble bloqueo del sistema renina angiotensina aldosterona. Todavía no sabemos si estas diferencias se deben a las condiciones clínicas estudiadas o al tipo de IECA o ARAll utilizado.

Ver glosario*

Gabriel Waisman [ Servicio de Clínica Médica. Hospital Italiano de Buenos Aires. gabriel.waisman @ hospitalitaliano.org.ar ]

Recibido el 5/5/08 y aceptado el 11/05/08

Waisman G. Telmisartán, ramipril y su combinación tienen similar eficacia en pacientes de alto riesgo vascular. Evid. actual. práct. ambul; 11(3): 71, May-Jun.2008. Comentado de: The Ontarget Investigators. Telmisartan, Ramipril, or Both in Patients at High Risk for Vascular Events. N Engl J Med 2008; 358(15):1547-59. PMID: 18378520.

\section{Referencia}

1. Yusuf S y col. Effects of an angiotensin-converting-enzyme inhibitor, ramipril, on cardiovascular events in high-risk patients. N Engl J Med 2000;342:145-53.

2. Pfeffer M y col. Valsartan, captopril, or both in myocardial infarction complicated by heart failure, left ventricular dysfunction, or both. N Engl J Med 2003; $349: 1893-906$. 3. Dickstein $\mathrm{K}$ y col. Effects of losartan and captopril on mortality and morbidity in high-risk patients after acute myocardial infarction: the OPTIMAAL randomised trial. Lancet 2002;360:752-60.

4. Cohn J y col. A randomized trial of the angiotensinreceptor blocker valsartan in chronic heart failure. N Engl J Med 2001; 345:1667-75.

5. McMurray $\mathrm{J}$ y col. Effects of candesartan in patients with chronic heart failure and reduced leftventricular systolic function taking angiotensin-convertingenzyme inhibitors: the CHARM-Added trial. Lancet 2003;362: 767-71.

6. McMurray J y col. Relationship of dose of background angiotensin-converting enzyme inhibitor to the benefits of candesartan in the Candesartan in Heart failure: Assessment of Reduction in Mortality and morbidity (CHARM)- Added trial. Am Heart J 2006; 151:985-91. 